\title{
Horizons/Théâtre
}

Revue d'études théâtrales

10-11 | 2017

Genre et arts vivants

\section{Femmes herculéennes au music-hall : déconstruction d'un cliché}

\section{Nathalie Coutelet}

\section{(2) OpenEdition}

Journals

Édition électronique

URL : http://journals.openedition.org/ht/581

DOI : 10.4000/ht.581

ISSN : 2678-5420

Éditeur

Presses universitaires de Bordeaux

\section{Édition imprimée}

Date de publication : 1 juillet 2017

Pagination : 260-279

ISSN : 2261-4591

\section{Référence électronique}

Nathalie Coutelet, «Femmes herculéennes au music-hall : déconstruction d'un cliché », Horizons/ Théâtre [En ligne], 10-11 | 2017, mis en ligne le 01 juillet 2018, consulté le 21 juillet 2019. URL : http:// journals.openedition.org/ht/581; DOI : 10.4000/ht.581

\section{(c) (†) $\odot$}

La revue Horizons/Théâtre est mise à disposition selon les termes de la Licence Creative Commons Attribution - Pas d'Utilisation Commerciale - Pas de Modification 4.0 International. 


\section{Nathalie Coutelet}

Nathalie Coutelet est maîtresse de conférences au département Théâtre de l'Université Paris 8. Elle s'intéresse principalement à l'histoire des spectacles des $\mathrm{IIX}^{\mathrm{e}}$ et $\mathrm{XX}$ e siècles, notamment aux formes dites "populaires » telles que le cirque et le music-hall, mais aussi aux notions de démocratisation du spectacle et d'altérité.

Mail : nathalie.coutelet@univ-paris8.fr

Résumé : Si les numéros de force font partie intégrante de la tradition des foires et des cirques, leur appropriation par les femmes, à la fin du XIXe siècle, modifie profondément la pratique et, plus encore, la réception de cette forme. Associée à la notion de virilité, elle se métamorphose et offre un miroir des modifications sociales en cours et des luttes pour l'égalité.

Leona Dare ou Miss Lala, ainsi, n'ont pas seulement rivalisé de force avec des acrobates masculins, mais renversé les rôles dévolus aux hommes et aux femmes, tant dans le

Abstract: If lift-acts are an integral part of the tradition of fairs and circuses, their appropriation by women at the end of the $\mathrm{XIX}^{\text {th }}$ century, deeply changes the practice and, beyond, its reception. Associated to the notion of virility, this form is transformed by women and offers a mirror of ongoing social changes and struggles for equality.

Leona Dare or Miss Lala not only competed with masculine acrobats, but also inverted the roles assigned to the men and to the women, in the shows and in life. Looking at their acts implies more than an aesthetic spectacle que dans la vie. L'appréhension de leurs numéros n'est donc jamais uniquement esthétique, mais toujours conditionnée à leur statut de femmes.

Mots-Clés : numéros de force féminins, cirque, music-hall, virilité

approach, a comprehensive understanding of their status as women.

KeYwords: female strength's routines, circus, music-hall, masculinity 


\section{Femmes herculéennes au music-hall : déconstruction d'un cliché}

Dès le XVIII ${ }^{\mathrm{e}}$ siècle, les foires ont hébergé les spectacles les plus hétéroclites, exhibitions animales et humaines, hercules, avaleurs de cailloux ou de sabres. Elles familiarisent le public avec les tours d'adresse d'hommes forts qui, au XIX ${ }^{\mathrm{e}}$ siècle, se présentent avec un « habit associant la peau de tigre à la massue $^{1} \gg$. Cependant, à la fin des années 1870 , des femmes supplantent les hommes dans ces numéros de force, qui font les beaux jours des music-halls. Le simple fait d'être exécutés par des femmes renforce le côté extraordinaire et spectaculaire de ces numéros. Ils accompagnent l'évolution sociale des femmes et les revendications féministes, mais se situent dans un contexte d'exacerbation de la « virilité » comme valeur centrale, de la pudibonderie incarnée par le sénateur Bérenger ${ }^{2}$, de la hantise de l'hystérie et de la dégénérescence.

Les numéros de force féminins, alliance des contraires entre une supposée force masculine et une tout aussi supposée grâce féminine, troublent le public en dérangeant ses codes, tout en le séduisant par l'aspect sulfureux qui leur est associé. Les deux premiers numéros de ce type, créés par Léona Dare et Miss Lala, permettent d'aborder la façon dont ces exercices déconstruisent les clichés en vigueur sur la féminité et la masculinité, comme le lien qu'ils entretiennent avec le contexte social, scientifique et culturel. Symboles d'un renouvellement des pratiques spectaculaires, ils concrétisent - souvent de façon involontaire - les luttes féministes contemporaines.

\section{Prologue : Les numéros masculins}

Pour mieux comprendre l'effet détonant produit par les numéros féminins, il faut connaître leur fonctionnement classique et originel, avec les artistes masculins. Les exercices de force réalisés par les « hercules de foire » constituent, en effet, un topos du spectacle music-hallien. Dans la lignée mythologique, ils font montre d'une force hors-norme : «enlèvement de poids » selon l'expression utilisée à l'époque, soulèvement de colonnes humaines, torsions de barres en sont les démonstrations les plus courantes. 
Leur physionomie et leur costume sont codifiés depuis le XVIII ${ }^{\mathrm{e}}$ siècle : moustaches, justaucorps moulant qui laisse percevoir les muscles, biceps saillants.

Leur succès répond au déploiement de la virilité comme vertu principale du XIX ${ }^{e}$ siècle ${ }^{3}:$ l'homme doit apparaître comme fort, dominant, énergique, que ce soit au travail, à la guerre, dans les duels, dans le lit conjugal ou au bordel. À la fin du siècle, l'un des plus célèbres hommes forts du music-hall est Napoli, capable de soulever un cheval et de porter un canon sur l'épaule. Joe Holtum, surnommé « l'homme aux boulets de canon » exécute, dit-on, des « miracles de force et d'audace ${ }^{4} \gg$. Aux sceptiques qui pensent à une supercherie, il offre « de donner trois mille francs à celui qui, comme lui, recevrait le boulet de pied ferme ${ }^{5} \gg$. Jack de fer, lui, porte à bout de bras un cheval, puis, en fin de numéro, un piano à queue et les musiciens, groupés sur une plateforme ${ }^{6}$. Cependant, la loi commerciale du music-hall oblige à renouveler sans cesse les prouesses ; des femmes créent donc l'événement en inaugurant l'ère des numéros de force féminins au music-hall, si bien qu'un chroniqueur écrit, en 1879 : « Les prix Montyon récompensent seulement la force morale des femmes et non leur force physique, ce qui est vraiment malheureux ${ }^{7}$.

Sous couvert d'ironie, on peut percevoir l'irruption des exercices musculaires féminins comme perspective d'une sortie des femmes du seul champ de la « vertu » domestique, une prise de pouvoir féminine, ne seraitce que dans le champ restreint des numéros de force.

\section{Leona Dare, une pionnière}

De son vrai nom Adeline Susan Stewart, Leona Dare est Américaine (1855-1922). Elle se produit pour la première fois en France en 1876, aux Folies-Bergère, mais est alors déjà célèbre aux États-Unis. Elle a épousé un acrobate, Thomas Dare, auquel elle emprunte son pseudonyme et qui est à l'origine de la création de son numéro ${ }^{8}$. 
Femmes herculéennes au music-hall : déconstruction d'un cliché




Leona Dare

New York Public Library's collection

Cette situation est classique dans l'univers du cirque et du music-hall, où des familles entières forment des troupes, qui ont toujours intégré des femmes. Ces dernières n'y souffrent pas des mêmes interdits que dans la « société civile » : il est considéré comme normal qu'elles travaillent, produisent un numéro, seules ou en groupe. Les débuts de Leona résument la façon dont son numéro sera perçu tout au long de sa carrière : « une superbe personne, aussi intrépide que gracieuse ; elle charme et effraye par sa hardiesse ${ }^{9} \gg$. Contrairement aux numéros masculins traditionnels, qui ne peuvent se renouveler qu'en décuplant encore les poids levés, celui de Léona métamorphose totalement la physionomie de l'exercice : sa féminité accentue la sensation de prouesse hors-norme et allie le plaisir de la surprise à la crainte des conséquences sur les statuts féminins et masculins : « Quand on la voit, suspendue en l'air, retenir avec ses dents le trapèze sur lequel évolue un vigoureux et souriant gaillard, on se demande lequel des deux appartient au sexe fort ${ }^{10}$. »

Ici, le phénomène réside dans la manière de tenir le trapèze - les dents autant que dans la féminité de l'artiste. On remarque que Leona ne surenchérit pas quant aux poids levés : ni cheval, symbole viril par excellence, ni piano ; elle n'utilise ni ses bras, ni ses biceps, eux aussi reliés symboliquement à la force musculaire masculine, mais ses dents. Au fond, les femmes ne sontelles pas socialement perçues telles des « croqueuses d'hommes » et Leona ne concrétise-t-elle pas cette séduction agressive et destructrice dans son numéro ?

Alors que les revendications féministes se développent, que la virilité est érigée en vertu cardinale d'une population que l'on veut protéger de la dégénérescence - surtout après l'humiliation de 1870 - le tour de force réalisé par Leona Dare déséquilibre les stéréotypes solidement ancrés et soigneusement entretenus. La proximité avec le danger et la mort est jugée comme une valeur virile; qu'une femme fraie avec ce danger est inhabituel, plus encore, inacceptable. Les détracteurs arguent du fait que cela contribue à 《viriliser » les femmes et à déséquilibrer le rapport masculin/féminin et une soi-disant « répartition naturelle » des rôles. Les médecins s'y intéressent d'ailleurs, l'époque étant attachée aux découvertes biologiques et anthropologiques : 
Le sexe à qui la nature refuse la force physique pour faire briller chez lui la grâce et la beauté des formes peut, au moyen de l'éducation, tromper les vues de cette même nature. Ainsi, de nos jours [... ], miss Leona Dare et plusieurs autres sont arrivées à la puissance herculéenne et à l'adresse gymnastique, sans rien perdre de la grâce qu'elles tiennent de leur sexe $e^{11}$.

La perturbation de ce qui est considéré comme l'ordre naturel des corps, constitue le cœur de l'attraction spectaculaire de Leona. Mais en conservant des caractères jugés « féminins », comme la grâce, elle devient une sorte d'hybride entre une force supérieure à la moyenne des hommes et une corporalité toute féminine. En d'autres termes, une figure androgyne, topos littéraire qui peuple alors les imaginaires ${ }^{12}$. Elle incarne donc cette crainte propre au tournant des $\mathrm{XIX}^{\mathrm{e}}$ et $\mathrm{XX}^{\mathrm{e}}$ siècles : l'émancipation féminine comme émasculation symbolique des hommes - émasculation au moyen des dents, une image on ne peut plus effrayante pour les hommes - une « virilisation » des femmes qui entraîne, par effet boomerang, l'effémination des hommes. D'ailleurs, le numéro de Leona Dare est un duo, dans lequel elle tient entre ses dents un trapèze sur lequel voltige... un homme. La femme, ordinairement élément voltigeur de l'acrobatie aérienne, renverse ici les rôles.

Hughes Le Roux, dans Les Jeux du cirque et la vie foraine ${ }^{13}$, évoque un proverbe circassien disant : « Si tu tiens à tes os, ne travaille jamais ni avec le vin, ni avec une femme » et le fait que « dans tout couple d'acrobates » il y ait « un mâle et une femelle, le héros fort, le porteur, en argot "l'homme du dessous", celui qui soutient le poids de tout l'exercice, celui auquel "l'homme du dessus" confie sa vie $\gg^{14}$. L'élément $\ll$ femelle $\gg$ est ici un homme, le voltigeur, celui qui est porté, jugé moins viril que le porteur, en l'occurrence Leona, la femme. Hughes Le Roux écrit pourtant bien après l'apparition des numéros de force féminins, ce qui en dit long sur leur caractère subversif pour le public et pour la critique.

Toutefois, les affiches des établissements ne montrent jamais le partenaire de Léona ; elle est toujours représentée seule, ce qui confirme bien que c'est elle l'héroïne du numéro, celle qui fera venir le public et qu'elle a, en quelque sorte, éliminé l'homme à son profit. Les jeux de mots sur les dents de Leona ont couvert les colonnes des journaux. Un article du Figaro narre la façon dont un dentiste a assuré sa publicité pendant les représentations de Leona Dare, afin de vanter la solidité des « rateliers » qu'il fabriquait ${ }^{15}$. En 1880, en tournée au cirque Renz de Hambourg, elle est victime d'un accident qui lui brise quatre dents et la presse s'inquiète, « puisque c'est dans ses mâchoires qu'elle trouve ses moyens d'existence $\gg$, mais rassure le 
lecteur en l'informant que « grâce aux soins du célèbre dentiste américain $\mathrm{X} . . . \gg^{16}$, elle a pu reprendre ses exercices. Cette fois, Leona devient objet commercial, support de stratégies « marketing » dirait-on aujourd'hui. Tout en égratignant l'incongruité d'un tel numéro pour une femme, ces articles confirment la récupération commerciale dont aucun ne veut se priver.

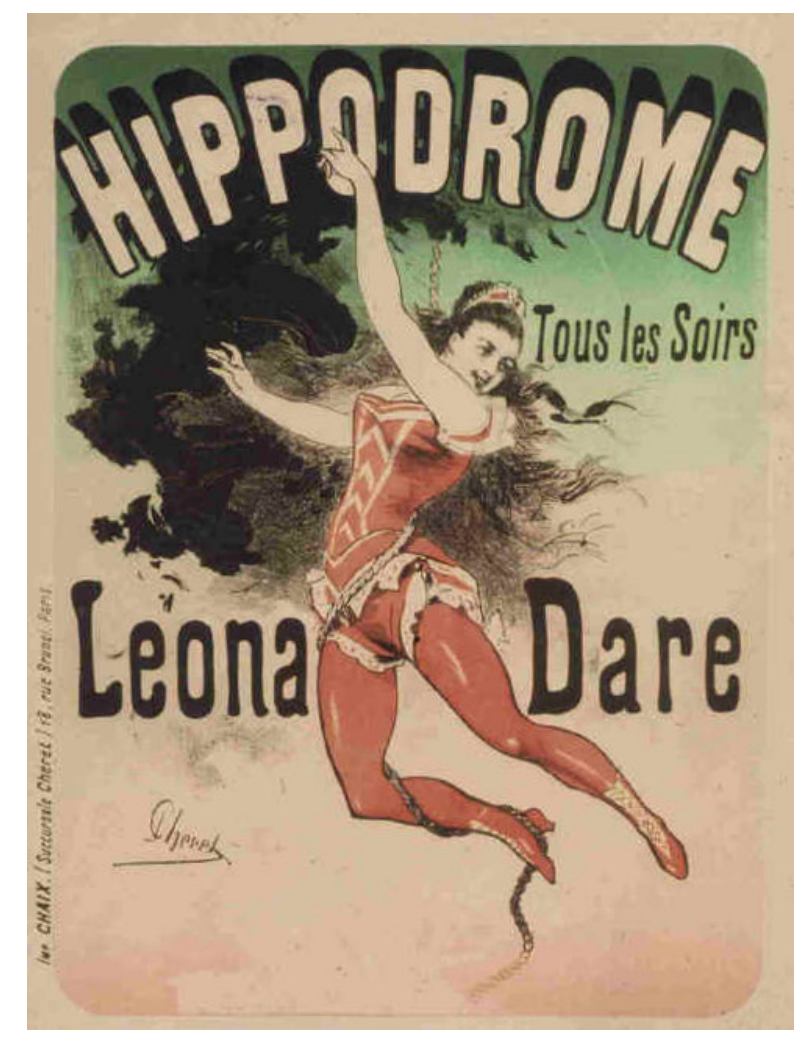

Leona Dare, affiche de L'Hippodrome par Jules Chéret, 1883.

Wikimedia Commons

La métaphore sexuelle qui émane de ce numéro n'échappe évidemment pas aux observateurs, d'autant que le partenaire n'est autre que son mari. Cette situation inédite du couple, fût-il circassien, laisse une impression mitigée au public. Le fantasme de dévoration par la femme qui nourrit la fin $\mathrm{du} \mathrm{XIX}^{\mathrm{e}}$ siècle trouve ici une nouvelle expression, qui provoque tout autant l'angoisse que l'excitation ${ }^{17}$. Toutefois, la masculinisation par la force 
de Leona Dare est contrebalancée par le fait qu'elle soit perçue telle « une fort jolie femme $\mathrm{e}^{18} \gg$, une $\left\langle\right.$ lionne ${ }^{19} \gg \ll$ aux formes plantureuses ${ }^{20} \gg$, aux « qualités plastiques ${ }^{21}$ », même si Alexandre Dumas fils aurait souhaité qu'après «ses exercices d'homme » elle « redev[ienne] une femme » en « saluant le public comme une française, avec toute la grâce qu'elle a » au lieu d'envoyer des baisers, à la mode américaine, pour réconforter les hommes qui l'applaudissent, alors qu' « elle vient [de les] humilier ${ }^{22}$ ».

Selon les affirmations de la presse de l'époque, chaque spectateur, par un phénomène d'identification, se sent personnellement diminué, menacé dans sa virilité23. Mais les affiches soulignent également son costume moulant, qui excite les regards masculins. Il est aux couleurs américaines et fait, à ce titre, souffler un vent de modernité dans les programmes français ; il rappelle pour nous celui de la super-héroïne Wonder Woman, univers au fond peu éloigné de l'« ovni »que représente Leona dans le spectacle de son temps. Enfin, le costume qui laisse paraitre ses formes, s'il correspond aux habitudes du music-hall, permet aussi de proposer au public, pour compenser si l'on veut la crainte liée à la surpuissance féminine, une vision érotique. Ses cheveux, ainsi, sont dénoués, ce que l'on ne fait pas en société et qui renvoie aux heures de l'abandon intime ; elle porte des chaussures à talons, certes peu vouées aux exercices de voltige, mais destinées à renforcer sa féminité, selon les codes en vigueur.

Une affiche des Folies-Bergère la montre les seins nus, sorte de Mercure féminin, nouvelle déesse des voyages aériens en trapèze ${ }^{24}$. Il semble improbable - la presse n'aurait pas omis de mentionner ce détail croustillant - qu'elle ait paru dans cette tenue ; il s'agit, là encore, d'une stratégie commerciale, typique du music-hall de l'époque, qui doit éclipser ses rivaux en surenchérissant constamment sur les chairs féminines dénudées dans le spectacle. L'affiche n'évoque absolument ni trapèze, ni tour de force et se concentre sur le corps de Leona, vêtue d'un slip, de spartiates ailées, d'une sorte de bandeau ailé sur les cheveux, tenant d'une main le caducée et levant l'autre vers deux miniatures, représentant son corps dans des postures sensuelles. Pour interpréter cette image, il faut recourir aux codes spécifiques du music-hall. Le XIX ${ }^{\mathrm{e}}$ siècle a affiché un goût certain pour les représentations mythologiques, qui permettaient, en art, de montrer le corps nu en le recouvrant d'un voile de légitimité classique. Le music-hall a fait son miel des thèmes antiquisants qui lui offraient un support de choix pour les scènes de bacchanales ou d'orgies, les tableaux vivants figurant des sculptures antiques et exhibant des postures destinées - officieusement bien sûr - à émoustiller le public. Toutefois, l'affiche peut fonctionner tel un avertissement : Mercure est 
le Dieu qui accompagne les âmes en enfer, manière de prévenir le spectateur de ce qui l'attend avec une femme dotée d'une telle puissance.

Les relations amoureuses de Leona pimentent encore son numéro. Le lecteur suit dans la presse les détails de sa vie privée - réelle ou construite de toutes pièces. En 1880, alors qu'elle se produit à Berlin, elle aurait causé un scandale en réunissant dans un dîner à sa gloire des dignitaires de la cour ${ }^{25}$. En 1882, à l'Hippodrome, son mari, Thomas Dare, fait du tapage, car il se meurt d'amour pour sa belle, qui l'a quitté pour un autre, Ernest Grünebaum, « jeune viennois », non majeur, dont le père intente un procès à Leona pour obtenir l'annulation du mariage ${ }^{26}$. L'année suivante (1883), la rumeur court que « deux gentlemen très correctement mis se sont boxés » pour «Mlle Leona Dare ». En 1897, on lui prête une relation avec Guillaume I ${ }^{\mathrm{er}}$, alors qu'elle se produit à Ems. Autrement dit, pour le public, la force de ses dents en fait, par essence, une croqueuse d'hommes.

Entre crainte face à cette surpuissance féminine et fantasme autour d'un corps tonique et musclé, c'est bien la sensualité qui semble triompher. La vision de «l'homme, accroché à son trapèze », tournoyant « entre les lèvres $\operatorname{roses}^{27}$ », de Leona Dare qui « vous tient un monsieur entre ces précieuses molaires sans avoirl'air de s'en apercevoir », si bien « que l'on se demande quel est le plus à plaindre, de la gymnasiarque ou du malheureux garçon dont le sort se borne à tournoyer toute la vie entre les dents de la jolie femme $e^{28} \gg$ résume l'état d'esprit de ce que Anne-Lise Maugue nomme « l'identité masculine en crise $\gg$, au tournant du $\mathrm{xx}^{\mathrm{e}}$ siècle $^{29}$. L'émancipation féminine, ô combien exacerbée dans ces numéros, se traduit par un sentiment de perte de repères masculins et une angoisse de dévirilisation, apparentée à une dégénérescence de la race, dans une époque vouée aux études anthropologiques. Ce dernier point est particulièrement sensible dans la réception des numéros présentés par Miss Lala. 


\section{Miss Lala, femme, Noire et herculéenne}

Miss Lala, de son vrai nom Anna Olga Brown, est née en $1858^{30}$, à Stettin, d'une mère allemande et d'un père afro-américain ou afro-caribéen, qui étaient artistes de cirque.

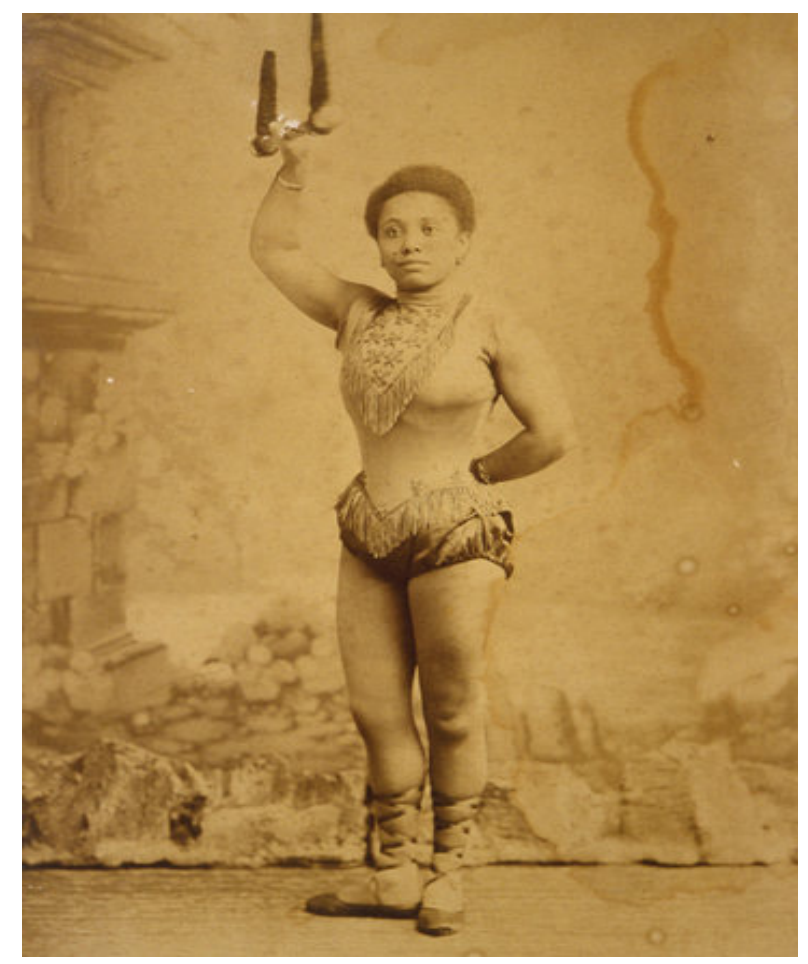

Miss Lala, 1880.

Wikimedia Commons

Son histoire, assez peu connue, a donné lieu aux versions les plus fantaisistes, comme celle d'une princesse africaine ayant perdu son trône, obligée de se reconvertir dans le spectacle; ou celle d'une femme vendue comme esclave se retrouvant dans un cirque du Sud de la France. Elle se produit dans un numéro assez proche de celui de Leona Dare, mais possède un atout supplémentaire : son métissage, traduit dans la presse et les affiches par les termes « la négresse » ou « la jeune mulâtresse ». On la désigne aussi régulièrement comme une «Amazone », symbole de la femme maîtresse de son destin, forte, à la fois désirable et menaçante ${ }^{31}$. En l'occurrence, l'expression 
peut également renvoyer aux célèbres Amazones de Behanzin, qui s'opposent farouchement à toutes les tentatives de colonisation européenne ${ }^{32}$.

En outre, alors que « la belle Leona ne portait qu'un homme, assez léger même, à l'aide de ses dents, Miss Lala en porte deux, en se balançant dans les diverses figures d'un trapèze animé33 $\gg$. Le fonctionnement circassien ou music-hallien nécessite, pour chaque artiste, de dépasser son prédécesseur et Miss Lala, qui se produit après Leona, doit réaliser un exploit supplémentaire. Peu de détails sont fournis au sujet de ces deux hommes, qui appartiennent vraisemblablement à la troupe Kaïra, avec laquelle elle se produit à cette époque. On se contente en général de noter qu'il s'agit d'une troupe «mixte », ce qui renvoie non à la parité hommes-femmes du groupe, mais aux origines ethniques différentes. L'autre différence apportée au numéro de Leona Dare est l'ajout d'un exercice inédit pour une femme : celui du canon. On lui met une pièce de canon de 300 kilos entre les dents et, suspendue par les jambes à son trapèze, elle soutient le choc. Sur l'affiche, le corps est ici mis en valeur ; la tenue colorée contraste soigneusement avec sa peau sombre ; elle porte des talons, une taille soulignée par la ceinture, un décolleté. La grâce traditionnelle de la voltigeuse est toutefois surprenante pour l'observateur, par la présence incongrue du canon au bout de ses mâchoires, dont le symbole phallique semble évident à tout observateur.

Décrite comme « une fille des Tropiques, au regard mobile, ardent ou langoureux. Petite, mais musclée comme l'Hercule Farnèse, pleine de grâce et de distinction ${ }^{34} \gg$, sa force musculaire la rapproche certes, du divin, mais surtout du masculin. De façon récurrente, les programmes et les coupures de presse la présentent surtout comme « la Vénus noire ${ }^{35} \gg$ ou $\ll$ la Vénus des Tropiques $^{36} \gg$. Ces appellations jouent bien évidemment sur l'intertextualité, puisque $\mathrm{La}$ Vénus noire, d'Adolphe Belot ${ }^{37}$ est publiée la même année et que Baudelaire a créé le type poétique de la « Vénus noire » dans les Fleurs du Mal. La littérature exotique et les exhibitions humaines ont préparé l'opinion publique aux sensations épicées procurées par les corps « autres ». L’orientalisme $^{38}$, dans le premier $\mathrm{XIX}^{\mathrm{e}}$ siècle, puis les découvertes liées aux récits des colonisateurs, propagés par les romans et les cartes postales, ont élaboré un type féminin dont l'animalité lui confère une sexualité débridée. Tandis que s'opère la hiérarchisation raciale, à grand renfort de mesures anthropométriques, la femme noire est présentée, comme figure littéraire, telle une femme dépourvue de tabous, soumise à son instinct sexuel vorace, favorisé par la chaleur du climat. Porter deux hommes par les dents, les « croquer » donc, renvoie à une émasculation, mais aussi à une forme d'émancipation. 
Que ce corps féminin noir domine par sa force deux hommes blancs ne lasse pas de générer un grand trouble. En cette période d'expansion coloniale, la virilité a été érigée en instrument de domination des populations, que l'on féminise pour mieux les dégrader : insistance sur les costumes, les bijoux, la pratique de la danse, etc. La phobie de la dégénérescence de la « race » (blanche) par l'efféminement est extrêmement forte et se concrétise d'une certaine manière dans ce numéro de force exécuté par une femme noire, dominant deux hommes blancs ${ }^{39}$. En même temps, les hygiénistes tendent à promouvoir le sport comme pratique indispensable de santé, la vigueur du corps comme vertu d'un individu sain. Le sport est instrumentalisé comme vecteur d'une virilité positive et, plus timidement, comme l'outil d'une perfection physique féminine : « Le meilleur résultat de la gymnastique et des exercices du corps, leur attrait le plus engageant, c'est de nous montrer combien leur pratique développe les belles formes ${ }^{40}$. 》

Miss Lala semble donc à la fois contredire et confirmer les théories de son temps, supérieure par sa force physique, inférieure par son statut de femme, inférieure parmi les inférieures en étant femme noire. Le Noir est en effet perçu à la fois comme inférieur sur le plan intellectuel et civilisationnel, mais, par sa plus grande proximité avec l'état de nature primitif et l'animalité, comme plus performant sur le plan physique (plus robuste, plus endurant, ce qui a aussi contribué à asseoir la politique esclavagiste). La femme est également considérée comme plus proche des pulsions primitives et animales, donc, une femme noire dotée de telles capacités physiques étonne, bien sûr, mais conforte aussi une certaine lecture des théories darwiniennes.

L'affiche montrant la troupe Kaïra est plus technique que celles qui vantaient les formes plantureuses de Leona Dare. Point de posture érotique ici, mais le corps au travail acrobatique. Le music-hall est fondé sur la présentation de numéros qui exacerbent une perfection surhumaine, plastique et technique. En ce sens, Miss Lala, comme Leona Dare avant elle, répondent aux objectifs habituels de ce type de spectacle. Mais, par leur féminité même, elles redoublent l'exploit. Miss Lala, enfin, surpasse sa consœur en apportant cerise sur le gâteau ou plume sur le spectacle - une altérité corporelle qui vaut à elle seule divertissement. D'ailleurs, toutes les affiches présentant le numéro de Lala assombrissent son corps et son visage, ce qui prouve bien que la couleur la place dans une altérité radicale, renforcée par la prouesse physique de ses exercices. C'est bien son nom qui est mis en vedette sur les 
affiches, tandis que ses partenaires sont anonymes, au sein de la mention générique « troupe Kaïra ».

En portant deux hommes blancs, Miss Lala suggère un trio amoureux dans lequel elle est la maitresse du jeu, mais aussi une « revanche » des populations colonisées et des femmes soumises aux libidos masculines. La « femme canon » telle qu'on la surnomme, laisse entendre une sauvagerie liée à ses origines et ravive le fantasme d'un homme se laissant dominer en amour. Contrairement au cliché largement construit par la littérature exotique et coloniale $\mathrm{du} \ll$ mariage colonial $\gg$, concubinage des colons blancs avec les femmes indigènes, Miss Lala domine les hommes, en général, les hommes blancs en particulier ${ }^{41}$.

Degas a immortalisé Miss Lala en 1879, après avoirvu son numéro au Cirque Fernando, alors que Leona a été régulièrement photographiée et a constitué le marronnier de la presse quotidienne, mais n'a pas retenu l'attention des plasticiens. C'est donc bien le corps féminin noir de Miss Lala qui fait d'elle une artiste exceptionnelle, peut-être plus encore que son numéro.

La toile montre un corps en costume blanc avec fanfreluches dorées, la tête vers le ciel, renversée, les dents mordant l'appareil relié au trapèze, les bras écartés. Tout son corps est en harmonie avec les lignes architecturales du toit du Cirque, mis en valeur par le vide du tableau, qui donne la sensation du vertige pour le spectateur. Car, comme le spectateur du Cirque, l'observateur de la toile voit le corps de Miss Lala par en dessous. Degas saisit le corps de l'artiste et en souligne la séduction, tout comme l'élévation, qui la rend supérieure, dans tous les sens du terme. L'intérêt plastique du peintre pour cette composition exprime également l'hybridité entre grâce, considérée comme « féminine » et force, considérée comme « virile ». Enfin, la posture de Miss Lala dans cette toile, tête renversée, bras écartés, jambes semi-pliées, peut suggérer un abandon corporel dans l'extase amoureuse d'un corps libre des tabous ${ }^{42}$. Les affiches assument leur vocation commerciale et doivent « vendre » le spectacle en pointant ce qui est susceptible d'attirer le public : une femme noire, portant grâce à ses dents, deux hommes blancs. Degas est intéressé, lui, par la plastique et le rapport des formes, des lignes, des couleurs.

La partenaire féminine de Miss Lala, Miss Kaïra, dont on apprécie le contraste chromatique, est davantage citée pour sa plastique avantageuse, alors que la véritable vedette est incontestablement Miss Lala. Éclipsée par la porteuse, Miss Kaïra est plus conforme aux critères de beauté du public ; la femme noire peut fasciner, voire susciter le fantasme secret, non être reconnue comme belle. 
Femmes herculéennes au music-hall : déconstruction d'un cliché

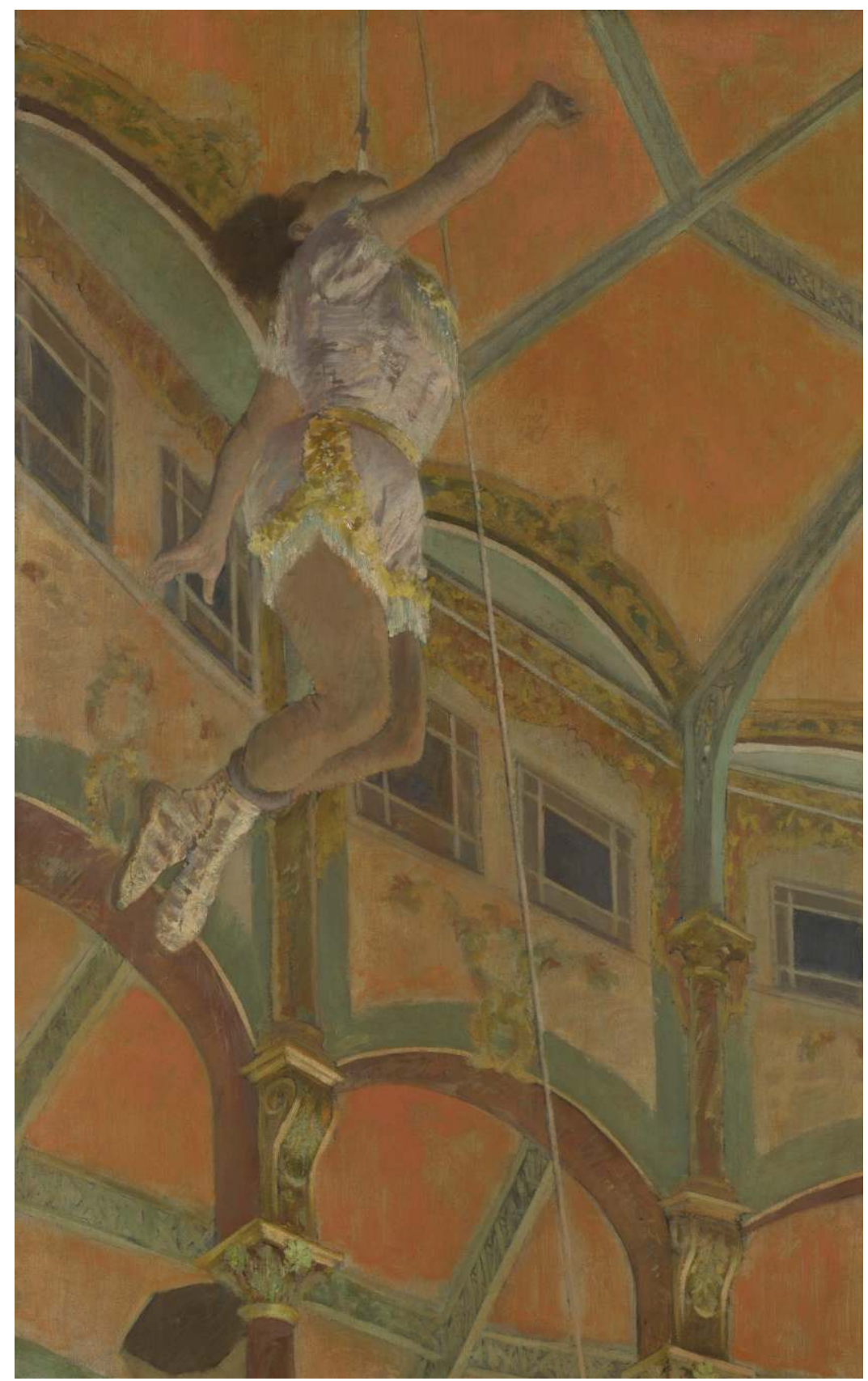

Miss Lala au Cirque Fernando, Edgar Degas, 1879.

Wikimedia Commons 


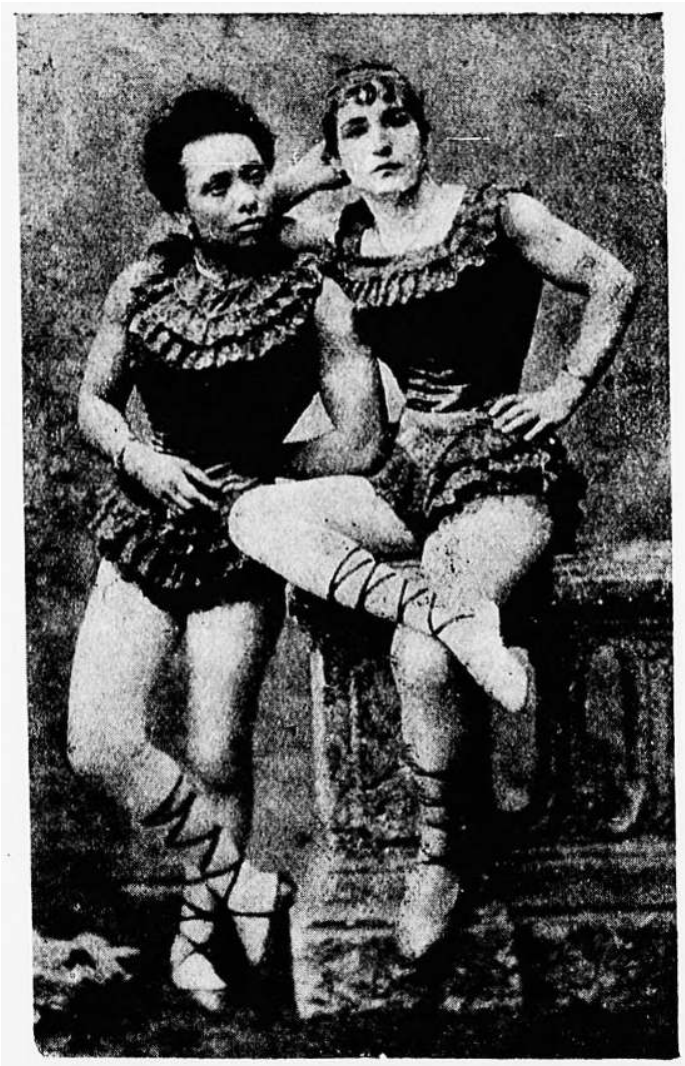

\section{Miss Kaïra et Olga}

Photo anonyme, dans La Culture physique, n 125, 15 mars 1910, p. 174, Gallica

Une certaine rivalité semble avoir émaillé leur collaboration, puisque, en 1879, toujours au Cirque Fernando, « pendant que miss Lala reposait sa mâchoire, Mme Kaira, désireuse de montrer la solidité de la sienne, s'était laissé $\left[\right.$ sic] hisser par les dents jusqu'au cintre du Cirque $^{43} \gg$ et est tombée, sans se blesser grièvement. À ce moment, les hommes ont disparu et ne restent en piste que les deux femmes : Miss Lala, « une négresse d'une force herculéenne ${ }^{44} \gg$ attrape au vol Mlle Kaïra, qui s'élance avec un saut périlleux à vingt mètres. Les reconfigurations des numéros, au gré des engagements des uns et des autres, sont tout à fait courantes. On ne peut tout de même s'empêcher de penser que les partenaires masculins ont fini par se lasser d'être les faire-valoir de ces dames qui leur volaient la vedette et les faisaient passer 
pour des sous-hommes. La presse, en effet, ne manque pas d'ironiser sur la domination implacable de femmes virilisées sur des hommes féminisés par leur apparence d'objets aux mains puissantes des artistes féminines.

Si les aventures amoureuses de Leona Dare avaient régulièrement alimenté les faits divers, Miss Lala n'est guère sujette à ce type de chroniques. Est-ce dû à sa couleur, qui permet de fantasmer à une union jugée « contre-nature », sans possibilité de la concrétiser et/ou de l'afficher ? À son corps noir, dont on peine à vanter les formes autres qu'athlétiques ? Toujours est-il que son mariage avec un Emmanuel Woodson, afro-américain, artiste de cirque, en 1888, et la naissance de leurs trois filles, qui deviendront elles aussi acrobates, la classent parmi les femmes « rangées », bien loin de la nymphomanie prêtée aux femmes artistes, en général, et aux femmes noires, en particulier.



Manuel Woodson, dit l'Amphisboëna

Photo anonyme, dans La Culture physique, n 125, 15 mars 1910, p. 174. 
Contrairement à Leona Dare, les critiques ne se sont pas concentrées sur son corps comme objet sexuel, mais comme force brute, et, à ce titre, admirée. Sa vision est nettement moins érotisée, dans les affiches comme dans les articles de presse. Elle incarne en revanche l'aliénation masculine tant redoutée en cette fin de siècle et une mainmise à la fois féminine et « nègre », ce qui engendre davantage de crainte que de désir.

\section{Conclusion}

Dans le trio Beretta, qui se produit à l'Olympia dans les années 1920, «c'était une jeune femme qui remplissait le rôle de porteur. Elle soutenait de son seul bras droit, levé verticalement, l'un de ses compagnons en équilibre de mains $[\ldots]$. Elle faisait plusieurs tours de scène en portant les deux athlètes sur ses épaules »; Miss Maggie Clifton, à la même époque, exécute son porté avec « une step-dance originale en tenant son partner en équilibre sur ses mains »; Agda, « habillée en gommeuse de caf'conc', servait de porteur aux deux autres artistes de la troupe », si bien que Gustave Fréjaville, grand spécialiste du music-hall, en concluait : « Il faut décidément ajouter aux récentes conquêtes du féminisme celle-ci : l'emploi de porteur dans les troupes acrobatiques ${ }^{45}$. $\gg$

C'est l'époque des « garçonnes $^{46} \gg$, qui amplifient la complexe réception des numéros perçus comme androgynes, où l'on aime voir une féminité exacerbée dans les courbes et le costume alliée à une force musculaire - sans hésitation sur le caractère féminin de l'artiste. C'était déjà le cas avec Leona Dare et Miss Lala, qui ont fait de leur corps féminin un atout, dont le costume ne laissait rien ignorer, tout en proposant des tours de force inédits pour des femmes. Elles ont été des incarnations de ces revendications féministes, malgré elles.

Certes, le cirque et le music-hall ne perpétuent pas les mêmes blocages que la société : les femmes y ont plus de visibilité et de légitimité. Cependant, on retrouve, dans la réception de leurs numéros, ces stéréotypes enkystés, mais bousculés par la nouveauté de leurs exercices. Leur travail de force excite l'imaginaire, force l'admiration, tout en heurtant les codes féminins et masculins. Autrement dit, ce que le spectateur - homme ou femme - peut admirer sans réserve au spectacle, il ne l'admettrait pas dans sa vie. Car, dans un contexte de dénatalité, de perturbation morale collective suite au traumatisme de Sedan et de la Commune, de revendications civiques féminines, la société vacille dans ses répartitions strictes des genres. Les numéros de force de ces femmes « herculéennes » contribuent, à leur 
manière, à interroger la répartition des rôles féminins et masculins, tout du moins dans l'univers circonscrit du spectacle vivant.

En tant que pratiques circassiennes et music-halliennes, ces numéros se placent dans la lignée des exercices toujours plus dangereux et nouveaux que les artistes doivent produire pour se faire remarquer. Qu'ils soient le fait de femmes, au fond, ne constitue qu'un attrait supplémentaire. Dans le champ social, en revanche, le caractère féminin de ces numéros constitue un razde-marée, qui submerge le public, partagé entre fascination et répulsion. Ces femmes, dont le corps peut susciter le fantasme érotique, ne sont pas les femmes que la société souhaite pour l'avenir ; mais elles représentent, vraisemblablement, une démonstration originale et magistrale de l'ineptie des clichés sur la force « virile ».

\section{Notes}

1. Sampion Bouglione-Marjorie Aiolfi, Le Cirque d'Hiver, Paris, Flammarion, 2002, p. 64.

2. Surnommé « le Père la Pudeur », objet de moqueries et de chansons caricaturales, René Bérenger est connu pour avoir pourfendu les mœurs jugées dissolues et pour avoir voulu abolir la prostitution.

3. Voir Alain Corbin, Jean-Jacques Courtine, Georges Vigarello (dir.), Histoire de la virilité, Paris, Seuil, 2011, tome 2.

4. «La soirée théâtrale », dans Le Figaro n ${ }^{\circ} 61,2$ mars 1875.

5. Gaston Vassy, «Informations », dans Le Figaro n ${ }^{\circ} 99,10$ avril 1875.

6. "Théâtres et concerts », dans Le Journal des débats politiques et littéraires, 20 février 1892.

7. Argus, «Chronique », dans La Semaine des familles n 21, 23 août 1879.

8. Leona Dare (1855-1922) épouse Thomas S. Hall, dit Thomas Dare, en 1871 à New-York. Voir Peta Tait, The Circus Bodies: Cultural Identity in Aerial Performance, London, Routledge, 2005.

9. Albert Laroque, « Revue des théâtres ", dans Le Petit Journal n 5101, 13 décembre 1876.

10. Ibid.

11. Géraud Marmisse, De l'Éducation physique, par le Dr Marmisse, Bordeaux, Imprimerie de J. Lamarque, 1877, p. 5.

12. Swendenborg a développé ce thème dans L'Amour vraiment conjugal (1768) ; au xIX siècle, Mademoiselle de Maupin de Théophile Gautier (1835), Séraphitus-Séraphita de Balzac (1834) illustrent cette tendance romanesque. Voir Frédéric Monneyron, L'Androgyne décadent. Mythe, figure, fantasmes, Grenoble, ELLUG, 1996.

13. Paris, Plon, 1889.

14. Ibid., p. 201. 
15. Un Monsieur de l'Orchestre, "La soirée théâtrale », dans Le Figaro n 193, 12 juillet 1878.

16. François Oswald, «Échos des théâtres », dans Le Gaulois n² 278, 17 juin 1880.

17. Voir Linda A. Davey, «La croqueuse d'hommes : images de la prostituée chez Flaubert, Zola et Maupassant », dans Romantisme n ${ }^{\circ}$ 58, 1987, p. 59-66.

18. Un Monsieur de l'Orchestre, «La soirée théâtrale », dans Le Figaro n 10, 10 janvier 1877.

19. François Oswald, «Échos des théâtres », dans Le Gaulois n 134, 26 janvier 1880.

20. Maurice Ordonneau, «La soirée parisienne », dans Le Gaulois n 932, 2 avril 1882.

21. Maurice Ordonneau, «La soirée parisienne. À l'Hippodrome », dans Le Gaulois n 964, 4 mai 1882.

22. Un Monsieur de l'Orchestre, "Courrier des théâtres ", dans Le Figaro n 259, 16 septembre 1877.

23. Voir par exemple "[elle] tient un homme suspendu entre ses dents, comme si c'était un paquet de plumes ». Un Monsieur de l'Orchestre, "La soirée théâtrale », Le Figaro, $\mathrm{n}^{\circ} 193,12$ juillet 1878.

24. Mercure, messager des dieux, est traditionnellement représenté avec un caducée, des sandales et un casque ailés ; Dieu des voyageurs, il accompagne les âmes en enfer.

25. François Oswald, «Échos des théâtres », dans Le Gaulois n 134, 26 janvier 1880.

26. Maurice Ordonneau, «Échos des théâtres », dans Le Gaulois n 64, 18 septembre 1882.

27. Un Monsieur de l'Orchestre, «La soirée théâtrale », dans Le Figaro n 193, 12 juillet 1878.

28. Maurice Ordonneau, «La soirée parisienne. À l'Hippodrome », dans Le Gaulois n ${ }^{\circ}$ 964, 4 mai 1882.

29. Anne-Lise Maugue, L'Identité masculine en crise au tournant du XXe siècle, 1871-1914, Marseille, Éditions Rivages, coll. « Histoire », 1987.

30. Consulté le 16 janvier 2017. URL : https://thecircusgirlblog.wordpress.com/tag/misslala/.

31. «Une amazone, c'est surtout une femme libre, vigoureuse, indépendante, musclée, qui vit par et pour elle-même. C'est le souvenir d'antiques peuples de femmes guerrières, dont on prétend qu'elles se coupaient un sein pour mieux tirer à l'arc. Pour le mouvement féministe, l'amazone peut être une source d'inspiration et de courage. Pour le sexiste mâle, c'est l'abomination de la désolation ", Pierre Samuel, «Les amazones : mythes, réalités, images », dans Les Cabiers du GRIF n 14-15, 1976, p. 10.

32. Les Amazones du Dahomey, vouées à la fidélité au roi Behanzin, dédient leur vie entière à la guerre. Vierges, elles apprennent le maniement des armes depuis l'adolescence et vouent une obéissance sans faille à leur souverain. Elles sont réputées être de féroces guerrières. Voir Stanley B. Alpern, Amazons of Black Sparta: the Women Warriors of Dahomey, London, Hurst, 1998.

33. "Cirque Fernando », dans L'Orchestre, décembre 1878.

34. Ibid.

35. "Programme des spectacles ", dans Le Figaro n ${ }^{\circ}$ 355, 21 décembre 1878.

36. Jules Prével, « Courrier des théâtres ", dans Le Figaro n 348, 14 décembre 1878.

37. Paris, Dentu, 1878. 
38. Voir Sarga Moussa, Sylvain Venayre (dir.), Le Voyage et la mémoire au XIXe siècle, Grâne, Créaphis, 2011 ; Edward Saï, L'Orientalisme. L'Orient créé par l'Occident, Paris, Seuil, 1980 (traduction Catherine Malamoud).

39. Voir Alain Corbin, Jean-Jacques Courtine, Georges Vigarello (dir.), Histoire de la virilité, op. cit.

40. "Cirque Fernando », dans L'Orchestre, janvier 1879.

41. Ce thème est, par exemple, développé dans Le Roman d'un Spahi, de Pierre Loti (1881). Voir Christelle Taraud, "Genre, sexualité et colonisation ", dans Sextant, Presses de l'Université libre de Bruxelles, 2008, n 23, p. 117-127.

42. Voir Marilyn R. Brown, "Miss la La's Teeth: Reflections on Degas and "Race” », dans The Art Bulletin, vol. 89, $\mathrm{n}^{\circ}$ 4, décembre 2007.

43. Jules Prével, « Courrier des théâtres », dans Le Figaro n² 299, 26 octobre 1879.

44. "Néron », dans Gil Blas n 1329, 9 juillet 1883. Le spectacle est donné à l'Hippodrome.

45. Gustave Fréjaville, Au Music-Hall, Paris, Éditions du Monde Nouveau, 1922, p. 149.

46. Voir Christine Bard, Les Garçonnes. Modes et fantasmes des Années Folles, Paris, Flammarion, 1998. Le terme de " garçonne ", toutefois, ne s'applique pas aux numéros de ces femmes que nous préférons nommer « herculéennes ", car elles allient caractères féminins exacerbés et force musculaire au-delà de la moyenne masculine. 